\title{
Concepción pedagógica para la enseñanza de lengua, centrada en la comprensión, análisis y producción del discurso
}

\author{
Pedagogical conception for the teaching of \\ language arts centered on the understanding, \\ analysis, and production of discourse
}

Ana Delia Barrera Jiménez

Universidad Nacional de Educación (Ecuador) ana.barrera@unae.edu.ec

Gisela Quintero de Chacón Universidad Nacional de Educación (Ecuador)

Luis Enrique Hernández Amaro Universidad Nacional de Educación

(Ecuador)

Nancy Marcela Cárdenas Cordero Universidad Católica de Cuenca (Ecuador)

Revista Cumbres Vol.3 №2

Versión impresa ISSN 1390-9541

Versión electrónica ISSN 1390-3365

http://investigacion.utmachala.edu.ec/revistas/index.php/Cumbres 


\title{
RESUMEN
}

El presente artículo tiene como objetivo fundamentar una concepción pedagógica, que favorezca la enseñanza de la lengua, centrada en los procesos de comprensión, análisis y producción del discurso, a partir de estrategias de intercambio académico entre la universidad y la escuela. Para ello, se realizó una investigación con un enfoque cualitativo, con énfasis en un estudio de carácter descriptivo, en la que resultaron clave los métodos del nivel teórico, empírico y estadístico utilizados, a partir de cuya aplicación se constataron limitaciones existentes en la Educación General Básica, en relación con el trabajo a favor de la lengua desde la perspectiva de una didáctica del habla, así como la falta de intercambio entre las instituciones y la universidad en pos de resolver tales insuficiencias. La concepción pedagógica propuesta articula el accionar de la universidad Nacional de Educación (UNAE) con las instituciones de la comunidad, en la que se precisan acciones que involucran los colectivos de asignaturas de lengua, la práctica pre - profesional y la educación continua, por lo que se arriba a la conclusión de cuan pertinente resulta que la universidad tenga un papel activo en dichas instituciones, en virtud de favorecer a la actualización de los docentes desde el punto de vista teórico y metodológico y con ello a perfeccionar el proceso de enseñanza aprendizaje que en ellas se lleva a cabo.

Palabras clave: Didáctica de la lengua, comprensión, análisis y producción del discurso.

\begin{abstract}
This article aims to support a pedagogical conception, which favors the teaching of language arts, centered on the processes of understanding, analysis, and production of discourse that resulted from the academic exchange developed between the university and the primary schools. For the achievement of this objective, a study with a qualitative approach was conducted. The study was mainly descriptive and was supported on the theoretical, empirical and statistical methods. These methods helped to unveil the limitations in the Basic General Education, in relation to the work in favor of the language from the perspective of a didactic of the speech. The absence of cooperation among primary schools and higher-level institutions as a mean to solve such insufficiencies was also found. The proposed pedagogical conception articulates the actions of the National University of Education (UNAE) with academic institutions of the community. Such actions require the involvement of language arts professors, prevocational training, and continuing education. It is concluded that the university has an active role in primary schools, by favoring the ongoing training of teachers from a theoretical and methodological point of view, contributing to the improvement of the teaching-learning process developed in such institutions.
\end{abstract}


Keywords: didactics of language arts, comprehension, analysis and discourse production.

\section{INTRODUCCIÓN}

La formación de los profesionales de la Educación constituye la misión y la visión fundamental de la universidad contemporánea, en la cual la Pedagogía del futuro se convierte en la principal prioridad. Ello implica que el proceso de formación, trascienda los límites cognoscitivos de las especialidades en particular, y se logren al término de cada una de las carreras, competencias imprescindibles para el desempeño exitoso del ejercicio de la profesión.

Lo anterior, presupone la consolidación de la Universidad como un espacio de desarrollo profesional por excelencia, derivado de la articulación de un pensamiento colectivo capaz de reflexionar conscientemente, en relación con un proceso formativo, que trascienda lo exclusivamente cognitivo y se proyecte hacia el logro de una verdadera integración de conocimientos, habilidades y actitudes, vinculadas a cada una de las áreas que complementan el proceso de profesionalización.

En este sentido, cobra una extraordinaria importancia, garantizar en los maestros en formación y en cada una de las carreras pedagógicas, capacidades para analizar, valorar, enjuiciar y fundamentar los procesos de comprensión y producción de significados como procesos nucleares del discurso oral, explicar la pertinencia de los recursos de la lengua en situaciones comunicativas orales concretas, así como para intercambiar con los demás y elaborar ideas en contextos concretos de comunicación oral y concebir un diálogo pedagógico eficiente en función del nuevo rol del maestro, como orientador y guía del proceso de enseñanza-aprendizaje.

Las capacidades antes referidas, hablan a favor de la necesaria competencia comunicativa en quienes eligen la profesión pedagógica, en función de lo cual la didáctica de la lengua como ciencia particular, asiste a cambios de concepción que, a partir de los avances de la Lingüística textual en las últimas décadas, dan fe del tránsito innegable de una lingüística de la lengua a una lingüística del habla y, en cuyo centro se reconoce al discurso, como representación más fehaciente del lenguaje en uso.

De esta manera, es necesario que la enseñanza de la lengua en los diferentes niveles educacionales, se centre en los procesos de comprensión, análisis y producción del discurso, lo que "deja atrás" la memorización de estructuras lingüísticas, su simple reconocimiento y, consecuentemente, la tradicional clase en la que el texto constituye solo un pretexto.

De acuerdo con las nuevas posiciones acerca del análisis del discurso expuestas por Gressler (2000), Van Dijk (2000), Beaugrande (2000), Roméu (2003), Barrera (2013), ente otros, la nueva perspectiva didáctica de la lengua defiende la aplicación del enfoque discursivo-funcional o descripción comunicativo-funcional del texto (Roméu, 2003), en aras describir y explicar la funcionalidad de los recursos lingüísticos en situaciones concretas de comunicación. 
Asumir las nuevas concepciones didácticas acerca de la enseñanza de la lengua, significa considerar tres componentes funcionales básicos: comprensión, análisis y construcción, entre los que se establece una relación de subordinación clave para concebir, coherentemente, las tareas que el profesor propondrá a los estudiantes. Estos componentes, según definición de Roméu (2011) en Barrera (2013) “... son los tres procesos esenciales que intervienen en la comunicación, y que los estudiantes deberán aprender a desarrollar, a fin de convertirse en comunicadores competentes" (pág. 102).

En esta dirección, urge reconsiderar desde la universidad, cómo enseñar a enseñar lengua, por lo que la Universidad Nacional de Educación (UNAE) como formadora de docentes en el Ecuador, juega un papel determinante en la preparación de los futuros maestros y, al unísono, en la búsqueda de alternativas para influir en el contexto objeto de la profesión, a partir de estrategias que articulen el trabajo al respecto, en el proceso de enseñanza-aprendizaje en los diferentes ciclos y en la Práctica pre- profesional, como integración de la teoría y la práctica.

A partir de lo anteriormente expuesto, se identificó el siguiente problema: ¿Cómo favorecer la enseñanza de la lengua, centrada en los procesos de comprensión, análisis y producción del discurso en la Educación Básica?

\section{Algunas consideraciones teóricas desde el punto de vista didáctico de la lengua}

El giro de las investigaciones lingüísticas, a partir de la década de los años 70 del pasado siglo, condujo al surgimiento de la lingüística del texto y el enfoque comunicativo. La lingüística del texto como ciencia aportó a los estudios lingüísticos un nuevo sistema de principios y categorías, en los que se destacan los trabajos de los funcionalistas ingleses acerca de la pragmática y el lenguaje en uso (Halliday, 1982; Austin, 1962); los representantes del estructuralismo en Norteamérica y Europa y los representantes de la semántica estructural francesa.

En las últimas décadas del siglo XX, el interés de muchos investigadores como Beaugrande (2000), Elosuá de Juan (2000), Escoriza (2006) y Barrera (2012), entre otros, se transfirió hacia una lingüística del habla o del discurso, con toda su complejidad y riqueza. Al respecto, se asume el lenguaje como medio esencial de cognición y comunicación social humana, como un sistema integrado con el conocimiento de los hablantes acerca del mundo y de la sociedad, que debe ser descrito "en términos lingüísticos, cognitivos y sociales, junto con las condiciones en las cuales lo usan los hablantes" (Beaugrande, 2000, pág. 74).

Tales definiciones, asegura Roméu (2013), apuntan a una concepción del lenguaje como un sistema múltiple, dinámico, diseñado para suministrar el medio para la comunicación humana. Su sustento teórico lo constituyen principios y categorías generales que sirven de basamento a los múltiples modos de orden práctico que se revelan en la diversidad discursiva.

El tránsito hacia una lingüística del discurso ha tenido su repercusión en la didáctica de la lengua, que ha comenzado a ocuparse de una enseñanza encaminada a revelar la relación entre lo que se significa, dónde tiene lugar 
el proceso de significación y mediante qué recursos lingüísticos se logra, de ahí que hoy hablemos también de una didáctica del habla o del discurso.

La enseñanza-aprendizaje de la lengua, por consiguiente, ha experimentado profundos cambios en las últimas décadas, al asumir las concepciones de la lingüística del texto y el enfoque comunicativo, lo que implica concebir la enseñanza centrada en los llamados componentes funcionales: comprensión, análisis y construcción de textos, y colocar en un primer lugar la enseñanza de estrategias que permitan lograr el dominio de dichos componentes.

Desde esta perspectiva, la enseñanza de lengua se caracteriza por la articulación entre los componentes funcionales: comprensión, análisis y construcción tetxual, cuyo tratamiento desde la clase responde a su jerarquización, de acuerdo con la connotación otorgada, a saber de priorizado o subordinado.

En este sentido, se considera como componente funcional priorizado, aquel que constituye el núcleo central de la clase, a partir del objetivo que se declara y en función del cual se estructuran los contenidos a tratar y se conciben las tareas docentes pertinentes. En correspondencia con ello, se concretan los restantes componentes didácticos del proceso de enseñanza-aprendizaje.

(...) entre el componente priorizado y el subordinado existe una estrecha relación, cuyo entendimiento es vital para concebir el proceso de enseñanza aprendizaje. Por tanto, la relación antes declarada, se convierte en "condición obligatoria" a tener en cuenta al realizar la dosificación de los contenidos de cada unidad del programa (concepción metodológica de los contenidos de la unidad con los profesores que imparten la asignatura en cada grado, atendiendo al tiempo de que disponen para el desarrollo de la unidad, objetivos a lograr, componentes que se trabajarán, tipo de clase, conceptos que se trabajarán, habilidades a desarrollar con la impartición de esos contenidos, actividades o ejercicios que deben realizar los estudiantes para lograr dichas habilidades y los medios de enseñanza a emplear). (Barrera, 2013, pág. 4).

Investigaciones desarrolladas por Roméu (2013) testifican que tanto la comprensión como la producción constituyen los dos polos del proceso de comunicación. Saber construir exige dar respuesta a las preguntas ¿qué significar? y ¿cómo significar?, lo que supone el conocimiento de la realidad (referente) y el dominio de habilidades constructivas del idioma: denominar (construir sintagmas nominales), predicar (construir sintagmas verbales predicados), elaborar (oraciones de diferentes grados de complejidad sintáctica), relacionar oraciones en torno a una idea temática (construir párrafos coherentes), relacionar párrafos referidos a un tema (construir discursos y textos extensos). Este proceso, reafirma, está relacionado con la intención comunicativa del que habla o escribe y tiene lugar en una situación comunicativa dada. La construcción de un texto está antecedida "obligadamente" de una adecuada comprensión del tema y de la situación comunicativa que lo sustenta, por eso se afirma que se subordina, en ese momento a la construcción.

Indisolublemente ligado al proceso de comprensión y construcción de significados, se halla el análisis del texto, mediante el cual el alumno descubre 
los medios empleados por el autor en su construcción, según los procedimien $\neg$ tos comunicativos de que se vale, según lo que trata de signifi $\neg$ car, su intención y finalidad.

El abordaje de estos componentes de manera interrelacionada y sistemáticamente en la clase de lengua obedece a una concepción didáctica que tiene en cuenta la relación entre la cognición, el discurso y la sociedad, misma que avala una nueva mirada al tratamiento de las estructuras gramaticales, o sea, al por qué la demanda, según Van Dijk (2000), de una gramática textual o comunicativa y que, según investigaciones realizadas por Barrera (2009) constituye una limitante didáctica, dado, entre otras razones, por la falta de actualización teórico-metodológica de los docentes..

La enseñanza de la lengua desde la posición que se defiende, destierra los métodos tradicionalistas que enfatizan en que el estudiante domine las estructuras lingüísticas, las identifique y reproduzca para que, en cambio, aprenda a explicar su funcionalidad en el discurso y su valor comunicativo y, consecuentemente, sea un comunicador eficaz. De esta manera, el proceso de enseñanza aprendizaje de la lengua se convierte en una vivencia en la que la atribución y producción de significados textuales constituye la principal vía para el desarrollo de las competencias requeridas desde el punto de vista comunicativo.

\section{MATERIALES Y MÉTODOS}

La investigación realizada responde al enfoque cualitativo, con énfasis en un estudio de carácter descriptivo, en aras de fundamentar la concepción que se defiende. Dicha investigación se corresponde con la línea de investigación de la UNAE número 4: "Didácticas de las materias curriculares y la práctica pedagógica".

Durante el proceso investigativo, se utilizaron métodos del nivel teórico (histórico-lógico; analítico-sintético; inductivo-deductivo; sistémico estructural), los que favorecieron el estudio de los referentes teóricos básicos relacionados con el tema y la estructuración de la concepción que se propone. Asimismo, del nivel empírico (encuestas, entrevistas y observación a clases), a partir de cuya información fue posible establecer, las tendencias predominantes en relación con el estado actual de la problemática que se investiga.

El uso de la estadística descriptiva, facilitó a partir del análisis porcentual de los resultados obtenidos determinar los indicadores más deficientes en relación con la temática investigada.

La unidad de análisis está constituida por 3 instituciones educativas de EGB del Cantón Azogues, en la que se realizó la práctica Pre-profesional de tercer ciclo de EB de la UNAE en el período abril - mayo de 2015.

Se aplicaron las encuestas y entrevistas a un total de 45 docentes que impartieran lengua, 15 en cada institución y a 12 directivos, 4 en cada centro. Se observaron un total de 30 clases de Lengua y Literatura: 6 de 2 do a 4to; 6 de 4 to a 6 to y 18 de $7 \mathrm{mo}$ a $10 \mathrm{mo}$, lo que permitió tener a una visión general acerca del tratamiento de la lengua en los diferentes grados de la EGB. 
Los instrumentos elaborados centraron la atención en:

- La concepción teórica y metodológica de los docentes de la EGB respecto a la enseñanza de la lengua.

- El nivel de prioridad otorgado a los componentes funcionales de la lengua en las clases.

- Rol de la universidad (UNAE) en el trabajo interactivo con las instituciones escolares, en función de perfeccionar el proceso de enseñanza-aprendizaje de la lengua.

- Prioridad investigativa a la didáctica de la lengua desde el trabajo científico-estudiantil en la UNAE.

En la entrevista a directivos se focalizó además el grado de interacción entre la UNAE y la escuela, en función del asesoramiento o capacitación como vía de perfeccionamiento del personal docente para la actualización didáctica dirigida a la enseñanza de lengua.

\section{RESULTADOS Y DISCUSIÓN}

Los resultados más sobresalientes a partir de la información diagnóstica, derivada del análisis de los instrumentos se muestran en la figura siguiente:

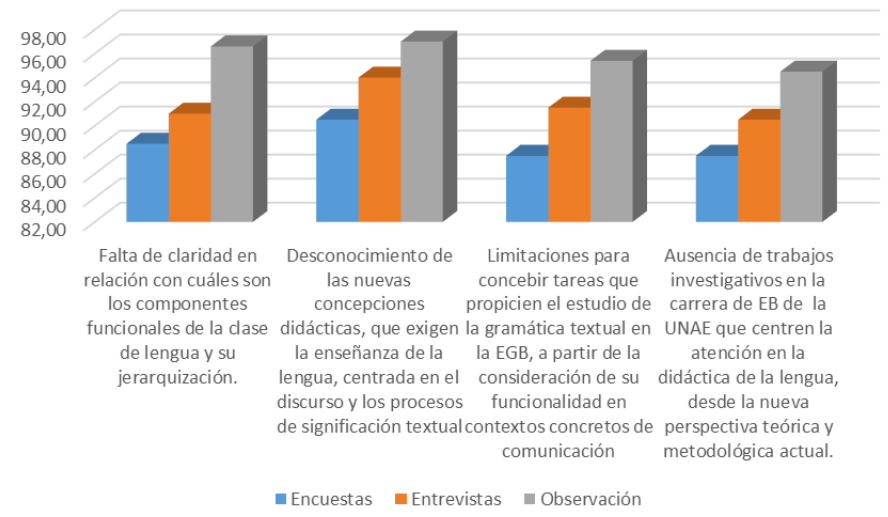

Figura 1: Insuficiencias detectadas

Los resultados obtenidos revelan que las insuficiencias detectadas son más evidentes en la observación, o sea, en lo que se hace en la práctica educativa. Resaltan las limitaciones en el conocimiento respecto a los componentes funcionales de la clase de lengua y, consecuentemente, su organización a partir de la requerida jerarquización, lo que afecta el tratamiento de la comprensión, análisis y construcción de significadas textuales. Ello se refuerza por el desconocimiento de las nuevas concepciones didácticas, que centran la atención en la enseñanza del discurso. En correspondencia, la gramática textual queda a la zaga ante un tradicionalismo que continúa imperando. Las limitaciones teóricas y metodológicas constatadas inciden en el alto porciento que revela la ausencia de trabajos investigativos en la carrera de EB, desde la nueva 
mirada didáctica de la lengua, lo que reclama de propuestas concretas al respecto.

Teniendo en cuenta los aspectos teóricos abordados y los resultados obtenidos en la aplicación de instrumentos referidos, se defiende una concepción pedagógica que garantice un trabajo conjunto entre la UNAE y las Instituciones de EGB, de modo que mediante las prácticas pre- profesionales y una estrategia de educación continua, los docentes de ambos contextos establezcan una red de intercambios de saberes y experiencias, respecto a la enseñanza de la lengua y las nuevas posiciones asumir.

\section{Acciones de la concepción pedagógica que se propone}

- Establecer sesiones de trabajo metodológico en la escuela, rectorado por un profesor de lengua de la UNAE, en los que se profundice en los referentes teóricos que sustenten una didáctica del habla.

- Realizar clases demostrativas por parte de profesores de lengua de la UNAE en las que se concrete el principio "teorizar la práctica", "practicar la teoría". (principio fundamental del Modelo de la UNAE).

Aplicar la Lesson Study en la escuela con la colaboración de los profesores tutores y los estudiantes vinculados a la institución durante el período de práctica profesional.

Establecer sesiones de análisis entre los docentes de la escuela, los de la UNAE que sean asignados como tutores, un profesor de Lengua y los estudiantes vinculados a la institución durante el período de práctica preprofesional, con el fin de valorar los resultados de la Lesson Study y realizar propuestas metodológicas concretas, a favor de la clase de lengua que se pretende en la EGB.

- Elaborar propuestas favorecedoras, respecto a la temática abordada desde los PIENSA en el tercer ciclo de la carrera de Educación Básica de la UNAE.

- Diseñar un Proyecto de Investigación, cuyo eje temático fundamental se identifique con la enseñanza de la lengua, centrada en los procesos de comprensión, análisis y producción del discurso.

- Concebir talleres de intercambio científico en los que se presenten propuestas innovadoras para el trabajo en función de una didáctica del habla.

- Socialización en la escuela de los resultados de los PIENSA, relacionados con didáctica de la lengua que se defiende.

- Establecer como línea desde Educación continua, la capacitación acerca de la enseñanza de la lengua desde la perspectiva defendida y a partir de ello, programar los cursos correspondientes.

La propuesta anterior ha sido valorada en el Colectivo de Comunicación de la UNAE, lo que ha derivado la toma de decisiones al respecto, que involucran a los profesores que lo constituyen. Asimismo, la concepción defendida forma parte del perfeccionamiento de la carrera de Educación Básica que se lleva a cabo en la UNAE en el presente período académico, en correspondencia con el currículo de la EGB. Por su parte, desde la Coordinación de posgrado se tiene en consideración la necesidad de lograr la comprensión de por qué 
concebir la enseñanza y aprendizaje de la lengua, desde la nueva perspectiva didáctica a la que se ha hecho referencia, lo cual será objeto de análisis en la Maestría en Educación en la mención Enseñanza aprendizaje de la Lengua y la Literatura, que se oferta la UNAE.

Igualmente, se considera un producto valioso derivado de esta investigación, el Proyecto PIENSA presentado en el ciclo anterior titulado: "El tratamiento de la gramática textual en la Educación Básica Superior. Una propuesta de Página Web, desde el proyecto de PIENSA"; así como artículos derivados relacionados con el tema, presentados en el II Congreso de la UNAE y prestos a presentarse en otros eventos internacionales

\section{CONCLUSIONES}

El estudio teórico y metodológico realizado, corrobora la pertinencia de una enseñanza de la lengua, centrada en los procesos de comprensión, análisis y producción del discurso, como concreción de la didáctica del habla.

La información diagnóstica obtenida, reveló insuficiencias en un mayor por ciento en cuanto la falta de claridad en relación con cuáles son los componentes funcionales de la clase de lengua y su jerarquización y al desconociendo de las nuevas concepciones didácticas, que exigen la enseñanza de la lengua, centrada en el discurso y los procesos de significación textual, lo que afecta la planeación de las tareas de aprendizaje relacionadas con la lengua desde una perspectiva actualizada.

La concepción defendida se centra en la articulación entre la universidad y las Instituciones educativas de la EGB, con énfasis en el trabajo metodológico, la demostración y rediseño de acciones en función de concebir una clase de lengua centrada en el discurso y los procesos de significación que le son inherentes.

\section{REFERENCIAS BIBLIOGRÁFICAS}

Austin, J. (1962). Palabras y acciones: Cómo hacer cosas con las palabras. Buenos Aires: Editorial Paidós.

Barrera. (2012). Coomprender y producir significados: Motivar el aprendizaje. España: Academia Española.

Barrera, A. (2013). Tratamiento metodológico de los componentes funcionales: Enseñanza de la comprensión de textos: Didáctica de la lengua Española y la Literatura. La Habana: Pueblo y Educación.

Beaugrande, R. D. (2000). El discurso como estructura y proceso. En T. van Dijk (Ed.), La saga del análisis del discurso (pp. 67-106 ). Barcelona: Gedisa.

Elosuá de Juan, M. (2000). Procesos de la comprensión, memoria y aprendizaje de textos. España: Sans y Torres, S. L.

Escoriza, J. (2006). Estrategias de comprensión del discurso escrito expositivo: Evaluación e intervención . Barcelona: Universitat de Barcelona.

Halliday, M. (1982). El lenguaje como semiótica social. México: Fondo de Cultura 
Económica.

Roméu. (2003). Teoría y práctica y análisis del discurso. La Habana: Pueblo y Educación.

Roméu, A. (2013). Didáctica de la Lengua y la Literatura I. La Habana: pueblo y Educación.

van Dijk, T. (2000). El discurso como estructura y proceso. En T. van Dijk (Ed.), El estudio del discurso (pp. 21-66). Barcelona, España: Gedisa.

\section{Cumbres}

